\title{
Fiebre botonosa mediterránea: A propósito de un caso
}

\author{
Álvarez Villacampa MB. ${ }^{1}$, Torres León JM. ${ }^{2}$, Menéndez Martínez Mª A. ${ }^{3}$, Casado Carreto MC. ${ }^{1}$, \\ Segovia Abad MaE. ${ }^{1}$
}

Sanid. mil. 2014; 70 (3): 185-187; ISSN: 1887-8571

\begin{abstract}
RESUMEN
Los síntomas típicos de la fiebre botonosa mediterránea (FBM) incluyen fiebre, un rash cutáneo generalizado y la presencia de una escara negra que puede pasar desapercibida. Por lo general sigue un curso benigno, aunque ocasionalmente aparecen complicaciones graves. A pesar de que la enfermedad fue descrita hace un siglo, algunas preguntas acerca del vector y el reservorio real de la enfermedad siguen sin aclararse. Presentamos un caso típico de FBM y planteamos una hipótesis sobre la forma de transmisión de la Rickettsia en nuestro paciente.
\end{abstract}

PALABRAS CLAVE: Fiebre botonosa mediterránea, Rickettsia conorii.

Mediterranean spotted fever: a case report

SUMMARY: Typical symptoms of Mediterranean spotted fever (FBM) include fever, generalized rash and the presence of a black eschar that may go unnoticed. Usually follows a benign course, but occasionally serious complications occur. Although the disease was first described a century ago, a few questions about the actual vector and reservoir of the disease remain unclear. We present a typical case of FBM and propose a hypothesis about the mode of transmission of Rickettsia in our patient.

KEY WORDS: Mediterranean spotted fever, Rickettsia conorii.

\section{INTRODUCCIÓN}

La fiebre botonosa mediterránea (FBM) es una zoonosis transmitida por garrapatas y causada por la bacteria Rickettsia conorii. En los últimos años, ha surgido un interés renovado sobre esta entidad por diferentes causas: la presencia de casos de FBM en regiones geográficas distintas a la zona endémica descrita por el propio nombre de la enfermedad, el descubrimiento de diferentes subespecies de Rickettsia conorii, la existencia de brotes de la enfermedad con un curso clínico complicado en un número elevado de pacientes o las hipótesis sobre los vectores y reservorio de la Rickettsia en la naturaleza. Presentamos el caso de un paciente con FBM y curso clínico favorable tras iniciar tratamiento antibiótico y se discuten algunos de los aspectos más novedosos de la enfermedad.

\section{OBSERVACIÓN CLÍNICA}

Varón de 71 años, que en Agosto de 2013 ingresó en nuestro Servicio por un cuadro febril acompañado de una erupción cutánea generalizada. El paciente tenía antecedentes de hipertensión

\footnotetext{
${ }^{1}$ MIR.

${ }^{2}$ Tte. Col. Médico.

${ }^{3}$ Médico civil. Hospital Central de la Defensa Gómez Ulla. Servicio de Medicina Interna. Madrid. España.
}

Dirección para correspondencia: Marcos Benigno Álvarez Villacampa. Servicio de Medicina Interna. Hospital Central de la Defensa Gómez Ulla. Glorieta del Ejército 1. 28047 Madrid. España. marcos.alvarez.villacampa@gmail.com

Recibido: 15 de octubre de 2013

Aceptado: 4 de abril de 2014 arterial, diabetes mellitus tipo 2 y dislipemia. Recibía tratamiento ambulatorio con candesartan, amlodipino, insulina glargina, metformina, sitagliptina y simvastatina. No tenía animales domésticos aunque era aficionado a la caza. Tres días antes de su ingreso comenzó con fiebre y odinofagia, fue tratado ambulatoriamente con amoxicilina-clavulánico. En los días sucesivos al cuadro febril se añadieron episodios de desorientación coincidentes con los picos febriles, astenia intensa, artromialgias, mal control de la glucemia y la presencia de una erupción cutánea. El paciente presentaba lesiones papulares eritematosas de menos de $1 \mathrm{~cm}$ de diámetro de predominio en la cara anterior del tronco y miembros (Fig. 1), existía afectación de palmas y plantas. En el glúteo derecho se apreciaba una lesión costrosa no pruriginosa de fondo negruzco y halo eritematoso (Fig. 2). En los estudios de laboratorio realizados a su ingreso destacaban leucocitosis leve con neutrofilia, glucemia de $400 \mathrm{mg} / \mathrm{dl}$ y creatinina de $1,6 \mathrm{mg} / \mathrm{dl}$, las concentraciones de transaminasas y el estudio de coagulación eran normales. Se dirigió la anamnesis, de forma intencionada, sobre la posibilidad de contactos recientes con animales. El enfermo nos refirió que había participado en una cacería de conejos en el mes de julio. Tras iniciar tratamiento con doxiciclina a dosis de $100 \mathrm{mg}$. orales cada 12 horas la fiebre desapareció y mejoró el estado general. El diagnóstico se corroboró en con un título positivos de IgM e IgG a Rickettsia conorii.

\section{DISCUSIÓN}

La FBM fue descrita, hace ahora un siglo, por Conor y Bruch en Túnez y, a partir de esta referencia, numerosos casos fueron declarados en países de la cuenca mediterránea. La escara típica de la inoculación, "la mancha negra", se reconoció en 1925 por 


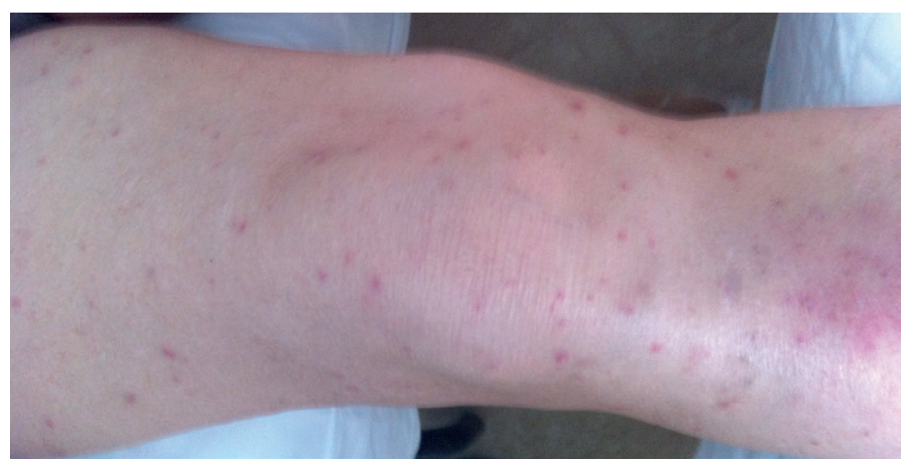

Figura 1. Lesiones papulares eritematosas en miembro inferior derecho.

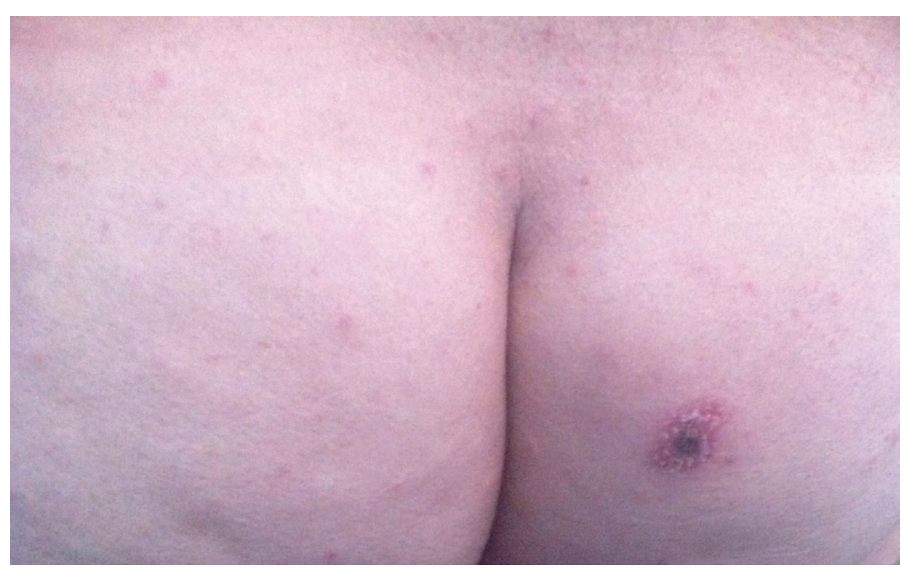

Figura 2. "Mancha negra" en el glúteo derecho del paciente.

Boinet y Pieri en Marsella. Unos años después, Durand y Conseil, demostraban la transmisión experimental del la enfermedad al hombre a partir de la garrapata Rhipicephalus sanguineus del perro y en el mismo año Blanc y Caminopetros observaban el paso transovárico en las garrapatas del agente productor de la enfermedad, de esta forma se planteó que la garrapata del perro era el vector y reservorio del microorganismo que causaba la FBM. El agente etiológico de la enfermedad, una bacteria Gram negativa del género Rickettsia que se comporta como parásito intracelular obligado, fue descubierto por Brumpt en 1932 que, en honor al autor de la primera descripción de la enfermedad, le dio el nombre de Rickettsia Conorii. En los últimos diez años, los avances en genética molecular han permitido conocer la secuencia genómica de algunas especies del género Rickettsia, entre ellas el de $R$. conorii, los resultados de estos estudios concluyen que aunque no existen diferencias genéticas suficientes para considerar nuevas especies de $R$. conorii si se debe considerar modificar la taxonomía con la distinción de varias subespecies ${ }^{1}$ : $R$. conorii conorii (responsable de la FBM), $R$. conorii caspia, $R$. conorii israelensis y $R$. conorii indica.

La FBM es endémica de la cuenca mediterránea, tanto del sur de Europa como del norte de África, pero también se han documentado casos en países del África subsahariana, como Sudáfrica, y en los que rodean el Mar $\mathrm{Negro}^{2,3}$. Existe una gran variabilidad en el número de casos por año, que en parte pueden ser explicados por una mayor actividad de las garrapatas en los años más calurosos ${ }^{4}$. La clínica que caracteriza la FBM es su- perponible a la descrita por Conor en 1910: un cuadro de inicio abrupto consistente en fiebre elevada, artromialgias, postración y la presencia al tercer o cuarto día de un exantema maculopapular generalizado que no respeta palmas ni plantas. La picadura de la garrapata suele pasar inadvertida, de forma que la "mancha negra" solo se pone de relieve tras la exploración física realizada con interés en su localización y a veces no llega a encontrarse.

La doxiciclina por vía oral (100 mg cada 12 horas durante 7-10 días) es el tratamiento de elección, su instauración precoz acorta el periodo sintomático. Pautas más cortas de $200 \mathrm{mg}$ cada 12 horas en un solo día pueden ser eficaces. En caso de intolerancia o alergia a tetraciclinas, la administración de ciprofloxacino (500 mg cada 12 horas durante 2 días) o cloranfenicol (500 mg cada 6 horas durante 7-10 días) es una alternativa. Las formas graves de la enfermedad pueden precisar corticoides y medidas de sostén ${ }^{5}$.

La tasa de mortalidad de la enfermedad es baja (1\%-3\%), sin embargo se han descrito diversas complicaciones que incluyen el fallo renal y cardiaco, flebitis o retinitis ${ }^{6}$. Los casos informados como graves muestran a veces una variabilidad estacional y geográfica que no se ha podido aclarar. En Salamanca durante 1984 el $19 \%$ de los casos sufrieron complicaciones frente a una tasa anual de complicación del $3.7 \%$ en los dos años anteriores ${ }^{7}$. En Beja, al sur de Portugal, la tasa de mortalidad en los pacientes hospitalizados fue del 32,3\% en $1997^{\circ}$. Diferentes hipótesis han intentado explicar estos hechos: la posible existencia de cepas de más virulentas de Rickettsia Conorii, los probables fallecimientos por cuadros febriles exantemáticos no diagnosticados en años anteriores como FBM, la comorbilidad añadida en algunos pacientes o la posibilidad de que otras especies de Rickettsias sean las responsables de los cuadros. Respecto a esto último, está probada la participación de Rickettsia conorii israelensis en algunos de los casos graves ocurridos en 1997 en Portugal ${ }^{5}$. En nuestro paciente las manifestaciones clínicas se acompañaron tan solo de un leve deterioro de la función renal que atribuimos a una baja ingesta hídrica y a un mal control de su diabetes.

Una cuestión debatida en la actualidad es la referida al reservorio de la enfermedad. La transmisión transovárica de $R$. conorii en la garrapata Rhipicephalus sanguineus es un hecho demostrado, sin embargo sólo una pequeña proporción de garrapatas están infectadas $(<15 \%)$ y estas suelen morir. Por otra parte, aunque Rhipicephalus sanguineus está presente en casi todo el mundo, la FBM se limita a determinadas regiones. Se ha llegado a postular que un mamífero jugara un papel como reservorio del agente causal de la enfermedad, en cuyo caso este animal debería cumplir unas condiciones que permitirían que las garrapatas se contagiaran tras picar a sus huéspedes: ser un huésped habitual del vector, ser susceptibles a las rickettsias y desarrollar una rickettsemia relativamente prolongada en el tiempo ${ }^{9}$. En el hombre no se cumplen estos criterios pues rara vez son infectados por un gran número de garrapatas y por otra parte la rickettsemia que se produce es de corta duración. Los perros son trasportadores de los vectores pero tampoco cumplen los requisitos para ser huéspedes definitivos pues al igual que en el hombre la rickettsemia es transitoria ${ }^{10}$. Desde las primeras descripciones de la enfermedad se ha atribuido a los conejos un papel en la trans- 
misión de la enfermedad y algunos hechos han reafirmado esta posibilidad: el descenso del número de casos de FBM en Francia coincidente con una epidemia de mixomatosis en 1952, el elevado número de conejos salvajes que presentan anticuerpos frente a Rickettsia conorii en España ${ }^{11}$, la elevada proporción de enfermos con FBM en algunas regiones de España que han estado en contacto con conejos $^{12}$ y la presencia de bacteriemia sin que estos animales se encuentren enfermos. En otros roedores como liebres y erizos pueden observarse características similares. En el caso que presentamos nuestro paciente refería haber estado en una cacería de conejos, si bien no podemos asegurar la forma que se produjo la picadura, una hipótesis factible es que el vector de la enfermedad fuera transportado por uno de los conejos cobrados y colocados por nuestro enfermo en una canana que llevaba en forma de cincha rodeando su cintura, el enfriamiento del cuerpo del animal provocaría el salto de la garrapata y su posterior picadura al cazador.

\section{BIBLIOGRAFÍA}

1. Zhu Y, Fournier PE, Eremeeva M, Raoult D. Proposal to create subspecies of Rickettsia conorii based on multi-locus sequence typing and an emended description of Rickettsia conorii. BMC Microbiol. 2005 Mar 14;5:11.

2. Pretorius AM, Jensenius M, Birtles RJ. Update on spotted fever group Rickettsiae in South Africa. Vector Borne Zoonotic Dis. 2004 Fall;4(3):249-60.
3. Parola P. Rickettsioses in sub-Saharan Africa. Ann N Y Acad Sci. 2006 Oct;1078:42-7.

4. Espejo Arenas E, Font Creus B, Bella Cueto F, Segura Porta F. Climatic factors in resurgence of Mediterranean spotted fever. Lancet. 1986;1:1333.

5. Merlo González V.E, Marcos Sánchez F, Árbol Linde F, Gómez Fernández M, Simón Martín A, López Onega P. Fiebre botonosa maligna. Med Intensiva 2002;26(1):21-3.

6. Leone S, De Marco M, Ghirga P, Nicastri E, Lazzari R, Narciso P. Retinopathy in Rickettsia conorii infection: case report in an immunocompetent host. Infection. 2008 Aug; 36(4):384-6. Epub 2007 Dec 14.

7. Ruiz Beltran R, Herrerro-Herrero JI, Martin-Sanchez AM, Vincente-Garcia V, Sanz-Ortega F, Mateos-Sanchez AA, et al. Formas graves de fiebre exantemática mediterránea. Análisis prospectivo de 71 enfermos. Ann Med Interna (Madrid). 1985;2:365-8.

8. De Sousa R, Nobrega SD, Bacellar F, Torgal J. Mediterranean spotted fever in Portugal: risk factors for fatal outcome in 105 hospitalized patients. Ann N Y Acad Sci. 2003;990:285-94.

9. Philippe Brouqui, Philippe Parola, Pierre Edouard Fournier and Didier Raoult. Spotted fever rickettsioses in southernand easternEurope. FEMS Immunol Med Microbiol. 49(2007)2-12.

10. Solano-Gallego L, Kidd L, Trotta M, Di Marco M, Caldin M, Furnanello $\mathrm{T}$, et al. Febrile illness associated with Rickettsia conorii infection in dogs in Sicily. Emerg Infect Dis. 2006;12:1985-8.

11. Ruiz Beltran R, Herrero-Herrero JI, Martin-Sanchez AM, Criado-Gutierrez LA. Role of lagomorpha in the wild cycle of Rickettsia conorii in Salamanca (Spain). Eur J Epidemiol. 1992;8:136-9.

12. Bartolomé J, Lorente S, Hernández-Pérez N, Martínez-Alfaro E, Marín-Ors A, Crespo MD. Clinical and epidemiological study of spotted fever group rickettsiosis in Albacete, Spain.Enferm Infecc Microbiol Clin. 2005 Apr; 23(4):194-6. 